\title{
Desigualdades socioeconómicas y mortalidad infantil en Bolivia
}

\author{
Edgar Maydana, ${ }^{1}$ Gemma Serral ${ }^{2}$ y Carme Borrell $^{3}$
}

Forma de citar Maydana E, Serral G, Borrell C. Desigualdades socioeconómicas y mortalidad infantil en Bolivia. Rev Panam Salud Publica. 2009;25(5):401-10.

RESUMEN Objetivo. Analizar las desigualdades socioeconómicas y su relación con la mortalidad infantil en los municipios de Bolivia en 2001.

Métodos. Estudio ecológico a partir de los datos del Censo Nacional de Población y Vivienda de 2001 para los 327 municipios de los nueve departamentos de Bolivia. La variable dependiente fue la tasa de mortalidad infantil (TMI) y las independientes fueron indicadores socioeconómicos indirectos (la proporción de analfabetos mayores de 15 años y las características constructivas y sanitarias de las viviendas). Se describió la distribución geográfica por indicador y se analizó la relación entre la TMI y los indicadores socioeconómicos mediante el coeficiente de correlación de Spearman y el ajuste de modelos de regresión de Poisson.

Resultados. La TMI estimada para Bolivia en 2001 fue de 67 por 1000 nacidos vivos. Las tasas fluctuaron entre < 0,1 por 1000 nacidos vivos en el municipio de Magdalena, Beni, y 170,0 por 1000 nacidos vivos en el municipio de Caripuyo, Potosí. La mediana de analfabetismo por municipio fue $17,5 \%$; la mediana de la proporción de viviendas que no tenían agua en su interior fue de 90,4\% y de las que carecían de servicios sanitarios fue de 67,6\%. La TMI se asoció inversamente con todos los indicadores socioeconómicos estudiados. Los valores de riesgo relativo $(R R)$ más elevados se observaron en las viviendas sin servicios sanitarios. En los modelos multifactoriales ajustados por el analfabetismo, los indicadores sin servicios sanitarios $(R R=1,50 ;$ IC95\%: 1,38 a 1,66), con paredes de adobe, piedra o tapial $(R R=1,54$; IC95\%: 1,43 a 1,67) y con techo de calamina, paja o palma ( $R R=1,34 ;$ IC95\%: 1,26 a 1,43) mantuvieron una fuerte asociación con la TMI.

Conclusiones. Se encontró una fuerte asociación entre una mala situación socioeconómica y una elevada TMI en los municipios de Bolivia en 2001. Los municipios de las zonas central y sudoriental del país presentaron la peor situación socioeconómica y las mayores TMI. El bajo nivel educacional, la ausencia de saneamiento básico y la precariedad de las viviendas, son factores claves que triplican el riesgo de muerte.

Palabras clave Mortalidad infantil, inequidad social, condiciones sociales, Bolivia.

1 Agència de Salut Pública de Barcelona; Universitat Autónoma de Barcelona; Agència de Gestió, de Ajuts Universitaris i de Recerca, Barcelona, España. La correspondencia se debe dirigir a Edgar Maydana, Agència de Salut Pública de Barcelona, Plaça Lesseps No. 1, 08023 Barcelona, España. Correo electrónico: emaydana@aspb.cat

2 Agència de Salut Pública de Barcelona, España.

Agència de Salut Pública de Barcelona; Universitat Pompeu Fabra, Barcelona; CIBER Epidemiología y Salud Pública (CIBERESP), Barcelona, España.
La relación entre las desigualdades sociales y la salud pública se comenzó a estudiar más profundamente desde hace tres décadas cuando un grupo de investigadores del Reino Unido analizó las desigualdades en la mortalidad, la morbilidad y el uso de los servicios de salud (1). Más adelante, otros estudios analizaron las desigualdades sociales en diversas zonas geográficas con el fin de ayudar a definir políticas y acciones es- pecíficas dirigidas a reducir las desigualdades en salud (2).

América Latina sufre de una enorme desigualdad. Aún en el país latinoamericano con menor inequidad en los ingresos hay más desigualdad que en cualquier país de la Organización de Cooperación y Desarrollo Económico (OCDE) —constituida por 30 países con altas rentas nacionales- o de Europa Oriental (3). El elevado nivel de desi- 
gualdad tiene costos considerables, como el aumento en los niveles de pobreza y la disminución de los efectos del desarrollo económico, destinados a reducirla, entre otros.

Bolivia es el tercer país más pobre de América Latina después de Honduras y Nicaragua; al menos seis de cada diez bolivianos viven con ingresos por debajo de la línea de pobreza (dos dólares estadounidenses, US\$). Bolivia presenta elevados índices de desigualdad: el ingreso medio del $10 \%$ más rico de la población es 45 veces mayor que el del $10 \%$ más pobre (4). De la población del país, $64,4 \%$ vive en situación de pobreza moderada y $41,5 \%$ en situación de pobreza extrema (55\% de la población rural y 22,3\% de la urbana). La pobreza se concentra de manera más notoria en los departamentos de Sucre y Potosí (5).

Según estudios realizados en Bolivia, las diferencias socioeconómicas abarcan los más diversos ámbitos, como el político, el laboral, el urbano, el económico, el étnico, el ambiental, el conductual de los habitantes y el relativo al saneamiento básico. El estado de salud de la población del país se caracteriza por una elevada morbimortalidad adulta e infantil, especialmente en las regiones rurales de mayor pobreza (6). De acuerdo con el Ministerio de Salud y Deportes, 60\% de la población de este país tiene menos de 25 años y solo $7 \%$ es mayor de 65 . La población boliviana crece a una tasa promedio anual de $2,7 \%$, con una tasa de fecundidad de 4,2 hijos por mujer (7). Más de la mitad de los bolivianos $(55,8 \%)$ se identifican a sí mismos como quechuas o aimaras y 6,1\% como guaraníes, chiquitanos, moxeños o miembros de alguno de los 32 grupos étnicos minoritarios de la amazonia boliviana (8).

El sistema de seguro nacional de salud boliviano está compuesto por tres sectores: el público, con programas priorizados dirigidos a las mujeres, los niños y los adultos mayores; el seguro social obligatorio -que puede ser de corto o de largo plazos-; y el seguro voluntario, que es privado. El seguro social obligatorio es administrado por las entidades gestoras de la seguridad social, cuyo órgano rector es la Caja Nacional de Salud (CNS), mientras que el seguro voluntario lo gestionan las administradoras de los fondos de pensiones (AFP), instituciones financieras privadas que administran la capitalización individual (9). Además del escaso sector privado, algunos segu- ros provistos por la Iglesia y otras entidades sin fines de lucro forman parte del seguro voluntario. Del total de la población, aproximadamente $30 \%$ tiene acceso a los servicios públicos de salud establecidos por los programas priorizados gratuitos, $27 \%$ mediante el seguro social obligatorio y $12 \%$ se atiende en servicios de salud privados; el restante $31 \%$ de la población no tiene acceso a la atención de salud por falta de recursos económicos y por no encontrarse en los segmentos cubiertos para los programas priorizados (10).

Aunque la mortalidad infantil está catalogada como un indicador de la calidad de vida, muchos países latinoamericanos tienen registros de eventos vitales poco desarrollados que no les permiten dar un seguimiento apropiado a estos indicadores. De acuerdo con datos de la Organización Panamericana de la Salud, en América Latina el subregistro de nacimientos es superior a $10 \% \mathrm{y}$, en el caso de Bolivia, el subregistro de la mortalidad es de $60 \%$, lo que dificulta el cálculo de la tasa de mortalidad infantil (TMI), comprendida como el número de defunciones en niños menores de 1 año por cada 1000 nacidos vivos en un determinado año.

Según Lavadenz y colaboradores, las tasas de mortalidad materna e infantil de Bolivia antes de 2001 estaban entre las más elevadas de América Latina: 370 por 100000 nacidos vivos y 67 por 1000 nacidos vivos, respectivamente (11). Pese a que el Estado, apoyado por entidades internacionales, ha impulsado algunos estudios sobre la desigualdad y la pobreza en Bolivia, no se cuenta aún con datos que relacionen los indicadores socioeconómicos con la TMI. El objetivo del presente estudio fue analizar las desigualdades socioeconómicas y su relación con la mortalidad infantil en los municipios de Bolivia en 2001.

\section{MATERIALES Y MÉTODOS}

Se realizó un estudio ecológico a partir de fuentes secundarias de información correspondientes al año 2001 para las 327 secciones municipales (municipios) de los nueve departamentos de Bolivia (figura 1). La población total del país en 2001 era de 8274325 habitantes, de ellos 204233 eran menores de 1 año (105 965 niños y 98268 niñas) (12). La media de habitantes por municipio era de 25 303,75 (mediana: 10 007; mínimo:
221 en el municipio de Yunguyo del Litoral, departamento de Oruro; máximo: 1131778 en el municipio de Santa Cruz de la Sierra, departamento de Santa Cruz).

El Censo Nacional de Población y Vivienda de 2001, realizado por el Instituto Nacional de Estadística (INE) de Bolivia, fue la principal fuente de información para estimar la TMI (variable dependiente) y las variables asociadas con las condiciones de vida de la población, apropiadas para medir los niveles de inequidad. El censo se realizó mediante entrevistas individuales a las personas mayores de 18 años de las viviendas habitadas (no incluyó a los que residían en cuarteles, hoteles, hospitales y otras instituciones), según el plan de sectorización nacional, departamental, municipal y de barrios elaborado por el INE (13). Esta información detallada del censo permitió establecer los municipios como unidad de análisis de este estudio.

\section{Variables e indicadores}

Para el estudio se clasificó la TMI en alta cuando era mayor de 30 por 1000 nacidos vivos, moderada si estaba entre 15 por 1000 y 30 por 1000 , y baja si estaba por debajo de 15 por 1000 . La información sobre el número de muertes se obtuvo a partir de las entrevistas censales, según la recordación de las mujeres en edad fértil, y se tomaron en cuenta únicamente los datos sobre nacimientos y muertes referidos al último año.

Para este estudio se calculó la proporción de analfabetos mayores de 15 años a partir del número de personas mayores de esa edad sin estudios (que no podían leer ni escribir o que habían abandonado los estudios antes de concluir el tercer año de primaria), con relación a la población total mayor de 15 años.

Otros indicadores socioeconómicos indirectos relacionados con la calidad de las viviendas tomados de los datos del censo del año 2001 y empleados en el presente estudio fueron los siguientes (porcentajes calculados a partir del número de viviendas con esas características con respecto al total de las viviendas censadas):

- con agua fuera de la vivienda: viviendas con abastecimiento de agua de fuentes comunitarias - ya fuera por cañería o no- que se encontraban fuera de la vivienda 
FIGURA 1. División política de Bolivia por departamentos

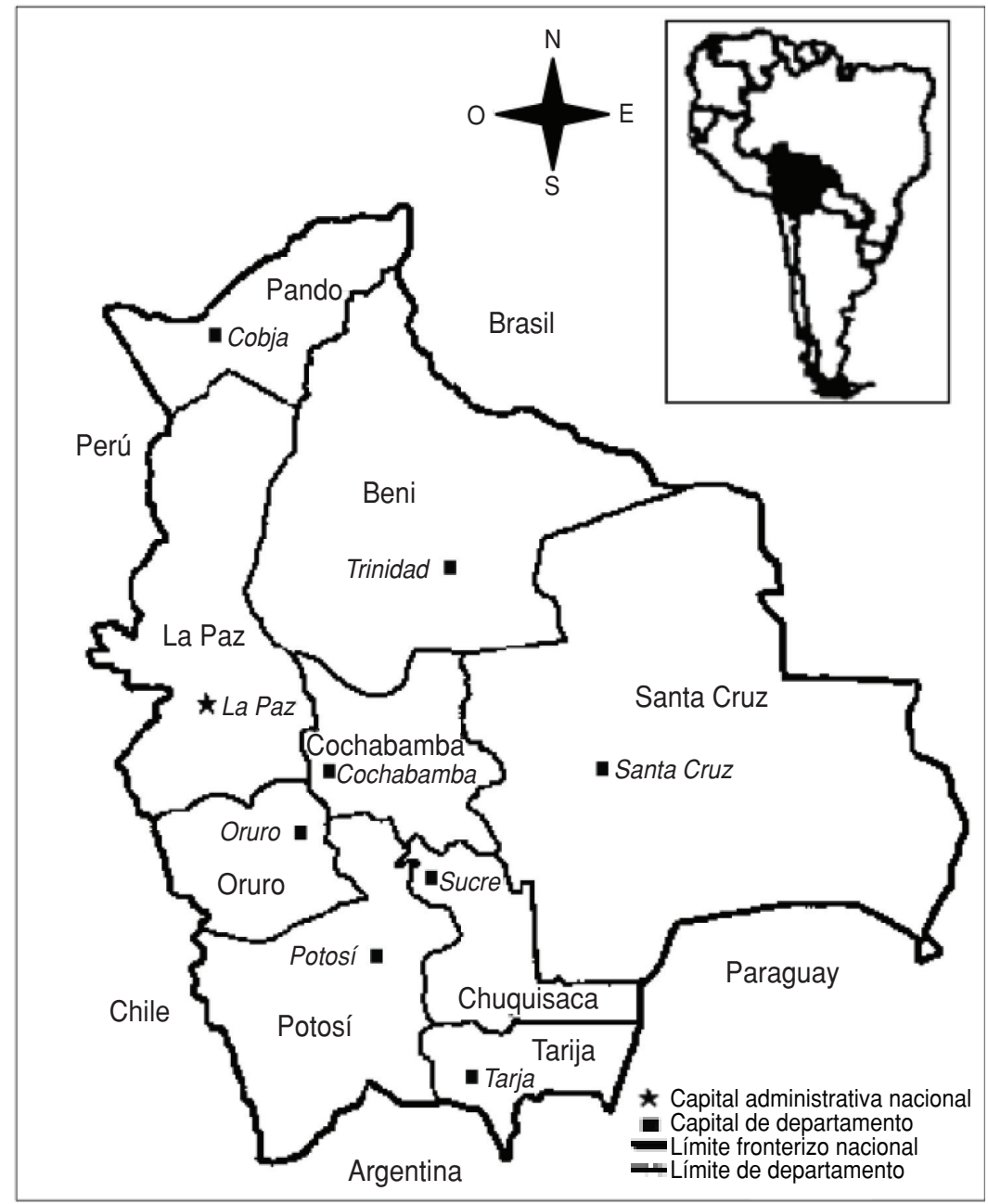

Fuente: creación propia.

- con agua de pila, pozo o río: viviendas que se abastecían de agua proveniente de piletas públicas o carros repartidores, pozos o norias sin sistema de bombeo, ríos, vertientes, acequias, lagos, lagunas o curiches (agua pantanosa estancada)

- sin electricidad: viviendas sin acceso al servicio de energía eléctrica

- sin servicios sanitarios: viviendas carentes de saneamiento básico para desechos humanos y aguas servidas

- con techo de calamina, paja o palma: viviendas con techo construido de calamina, plancha, paja o palma

- con piso de cemento, tierra o ladrillo: viviendas con piso de tierra, cemento bruñido o ladrillo

- con paredes de adobe, piedra o tapial: viviendas con paredes construidas con adobe, tapial, tabique, quinche, piedra, madera, caña, palma o troncos, tanto si las paredes interiores tenían revestimiento o no.

Con relación al material de construcción de las viviendas, los indicadores se refieren a los materiales principales utilizados.

\section{Análisis de los datos}

Los datos facilitados por el INE de Bolivia estaban agrupados por municipios, pero no por indicador. Se utilizó el programa informático SPSS versión 13.0 (14) para confeccionar la base de datos por municipio con todos los indicadores y una variable de referencia geográfica a partir de la latitud y la longitud.

Se empleó el paquete estadístico STATA versión 8.0 (15) para el análisis descriptivo de los indicadores por municipio; se calcularon los cuartiles 25, 50 y
75, la media, y los valores máximo y mínimo de las variables dependiente e independientes.

Mediante el sistema MapInfo versión 7.8 (16) se construyeron seis mapas nacionales con la distribución geográfica de las variables de estudio, desagregados por municipios, con el fin de facilitar la identificación territorial de los problemas socioeconómicos y de salud de interés.

Se analizó la relación entre la TMI y los indicadores socioeconómicos, así como entre los indicadores socioeconómicos entre sí, mediante los coeficientes de correlación de Spearman. Finalmente se ajustaron modelos de regresión de Poisson para calcular el riesgo relativo (RR) de mortalidad según las variables independientes. Como variable dependiente se utilizó el logaritmo de la TMI y como independientes se emplearon los indicadores socioeconómicos de los municipios categorizados en grupos separados por los cuartiles, así como el número de nacidos vivos, que se introdujo como una constante (offset). Se obtuvo un modelo para cada variable independiente y modelos multifactoriales para el análisis combinado de la TMI con el nivel de analfabetismo y cada uno de los otros indicadores socioeconómicos relacionados con las características de la vivienda. Para ajustar el exceso de dispersión encontrado en todos los modelos mediante las pruebas de verosimilitud, se empleó un modelo de cuasi-verosimilitud, equivalente a una distribución binomial negativa (gamma de Poisson). Este procedimiento permitió corregir el error estándar de las estimaciones de cada modelo (17).

\section{RESULTADOS}

\section{Distribución de la TMI}

A partir de los datos del censo, se estimó que en el año 2001 en Bolivia se registraron 13717 defunciones en niños menores de 1 año, para una TMI nacional de 67,0 por 1000 nacidos vivos. Por municipio, esta tasa varió entre un mínimo $<0,1$ por 1000 nacidos vivos en el municipio de Magdalena, departamento del Beni, y un máximo de 170,0 por 1000 nacidos vivos en el municipio de Caripuyo, departamento de Potosí (cuadro 1). En su conjunto, los valores más elevados se encontraron en el sudoeste del país (figura 2), en los departamentos de Chuquisaca, Cochabamba, 
CUADRO 1. Características de la población según los indicadores estudiados, por municipio, Bolivia, 2001

\begin{tabular}{lrrrrrrr}
\hline & & & \multicolumn{3}{c}{ Cuartil } & \\
\cline { 5 - 6 } \multicolumn{1}{c}{ Indicador } & Mínimo & Máximo & 25 & 50 & 75 & Media \\
\hline Población (habitantes) & 221 & 1131778 & 4931 & 10007 & 18516 & 25303,8 \\
Mortalidad infantil & & & & & & \\
$\quad$ Tasa por 1 000 nacidos vivos & 0,1 & 170,0 & 61,0 & 74,0 & 90,8 & 67,0 \\
$\quad$ Número de defunciones & 0 & 1202 & 8 & 18 & 40 & 42 \\
Analfabetismo en mayores de 15 años (\%) & 3,2 & 58,9 & 11,7 & 17,5 & 26,6 & 20,5 \\
Características de la vivienda & & & & & & \\
$\quad$ Con agua fuera de la vivienda & 34,8 & 100,0 & 81,9 & 90,4 & 95,2 & 85,9 \\
$\quad$ Con agua de pila, pozo o río & 5,5 & 98,2 & 35,1 & 56,7 & 73,5 & 54,9 \\
$\quad$ Sin electricidad & 4,7 & 100,0 & 50,6 & 71,1 & 89,5 & 66,6 \\
$\quad$ Sin servicios sanitarios & 4,6 & 99,3 & 35,2 & 67,6 & 83,7 & 59,8 \\
$\quad$ Con techo de calamina, paja o palma & 11,5 & 100,0 & 60,6 & 91,8 & 97,9 & 77,9 \\
$\quad$ Con piso de cemento, tierra o ladrillo & 4,1 & 100,0 & 91,1 & 96,4 & 98,6 & 91,6 \\
$\quad$ Con paredes de adobe, piedra o tapial & 6,0 & 100,0 & 72,5 & 94,8 & 98,4 & 81,0 \\
\hline
\end{tabular}

a Porcentajes calculados a partir del número de viviendas con esas características con respecto al total de las viviendas censadas.
Oruro y Potosí (71,0, 72,0, 82,0 y 99,0 por 1000 nacidos vivos, respectivamente). En los departamentos de Tarija, Santa Cruz y Beni — con menor grado general de privación socioeconómica- se presentaron las menores tasas.

\section{Distribución de los indicadores socioeconómicos}

La mediana del analfabetismo en mayores de 15 años fue de 17,5\%, con un valor mínimo de $3,2 \%$ en el municipio de
FIGURA 2. Distribución de las tasas de mortalidad infantil (TMI)a por municipio, Bolivia, 2001

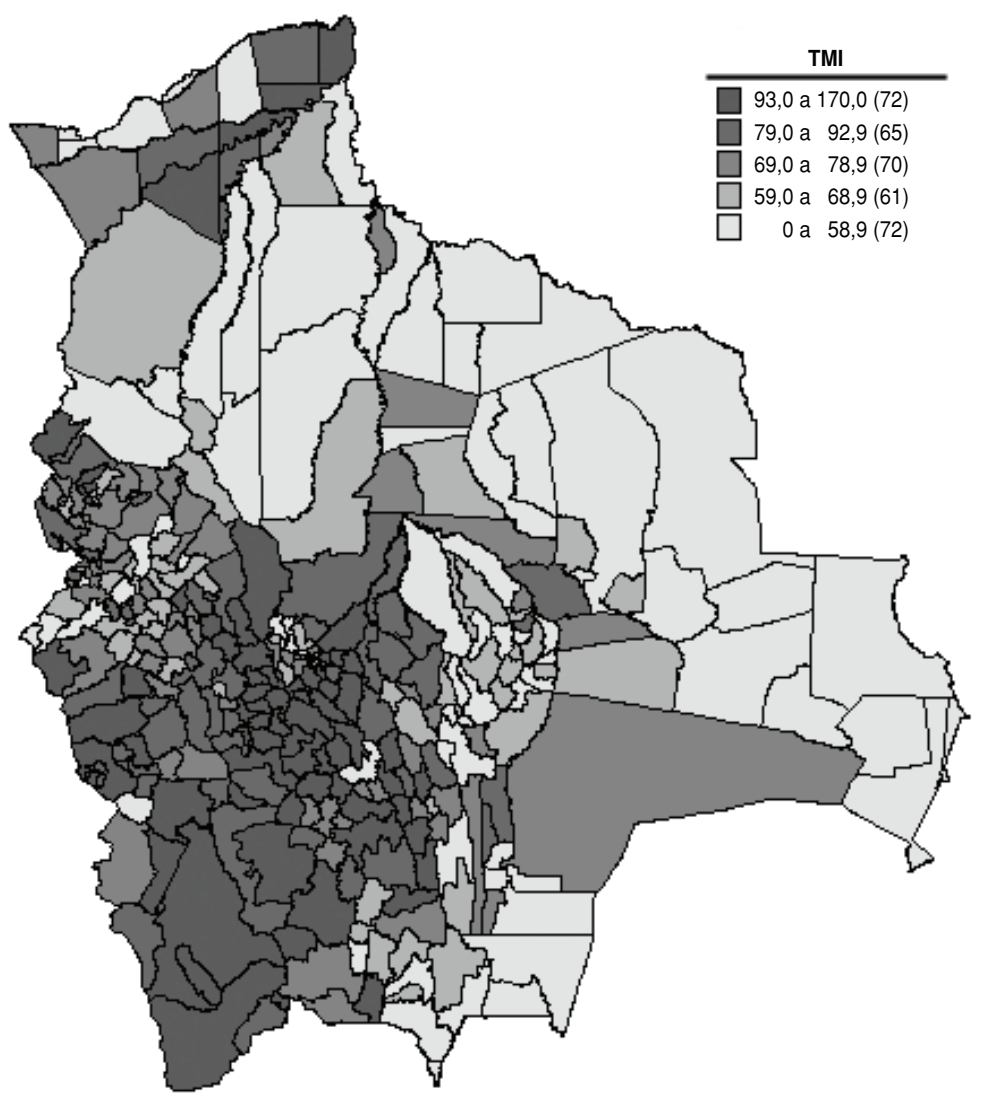

Fuente: A partir de los datos del Censo Nacional de Población y Vivienda de 2001 (9).

a Tasas por 1000 nacidos vivos. Entre paréntesis se ofrece el número de municipios clasificados en cada categoría.
Llica, departamento de Potosí, y un máximo de 58,9\% en el municipio Presto, departamento de Chuquisaca (cuadro 1). Las cifras más elevadas de analfabetismo se encontraron en los municipios del sur de La Paz, prácticamente todo el departamento de Oruro, el sur de Cochabamba, el norte y este de Potosí y todo el oeste de Chuquisaca (figura 3A).

Varios municipios tenían todas sus viviendas con condiciones desfavorables; el municipio de Entre Ríos, departamento de Tarija, fue el que tuvo una menor proporción de viviendas con agua fuera de ella $(34,8 \%)$. La mediana para este indicador fue de $90,4 \%$. Los cuartiles 25 y 75 mostraron cifras altas (81,9\% y $95,2 \%$, respectivamente), con una media de $85,9 \%$ para este indicador (cuadro 1).

El indicador más sensible en su asociación con la TMI fue "sin servicios sanitarios", con una mediana de $67,6 \%$. Se encontró una gran proporción de viviendas sin servicios sanitarios en casi todos los municipios de los departamentos de Potosí y Oruro, el sur de La Paz, el centro y el sur de Cochabamba y las tres cuartas partes de Chuquisaca (figura 3B), prácticamente coincidente con la distribución de la TMI (figura 2). A diferencia de otros indicadores, la brecha entre los cuartiles 25 y 75 casi se triplicó $(35,2 \%$ y $83,7 \%$, respectivamente) (cuadro 1); según los valores mínimo y máximo, entre $4,6 \%$ y $99,3 \%$ de las viviendas no contaban con este servicio.

Respecto al tipo de materiales predominantes en las viviendas, las medianas para los tres indicadores empleados fueron $91,8 \%$ (con techo de calamina, paja o palma), $96,4 \%$ (con piso de cemento, tierra o ladrillo) y 94,8\% (con paredes de adobe, piedra o tapial) (cuadro 1). Lo más representativo fue que las zonas con el indicador "con paredes de adobe, piedra, tapial" por encima del cuartil más desfavorable se ubicaron en una parte de Pando y las zonas del altiplano de La Paz, Oruro, Potosí y Chuquisaca, con cifras entre $98,7 \%$ y $100 \%$ (figura 3C). De esta forma, nuevamente en la franja occidental se concentraron las viviendas más precarias. También se encontraron municipios en los que la totalidad de sus casas tenían paredes de estos materiales (cuadro 1). Los valores de los cuartiles 25 y 75 fueron $72,5 \%$ y $98,4 \%$, respectivamente.

El indicador "con agua de pila, pozo o río" mostró una dispersión más notoria en las zonas central y occidental del país, 
FIGURA 3. Distribución de cuatro indicadores socioeconómicos ${ }^{\mathrm{a}}$ en los municipios de Bolivia, 2001

A

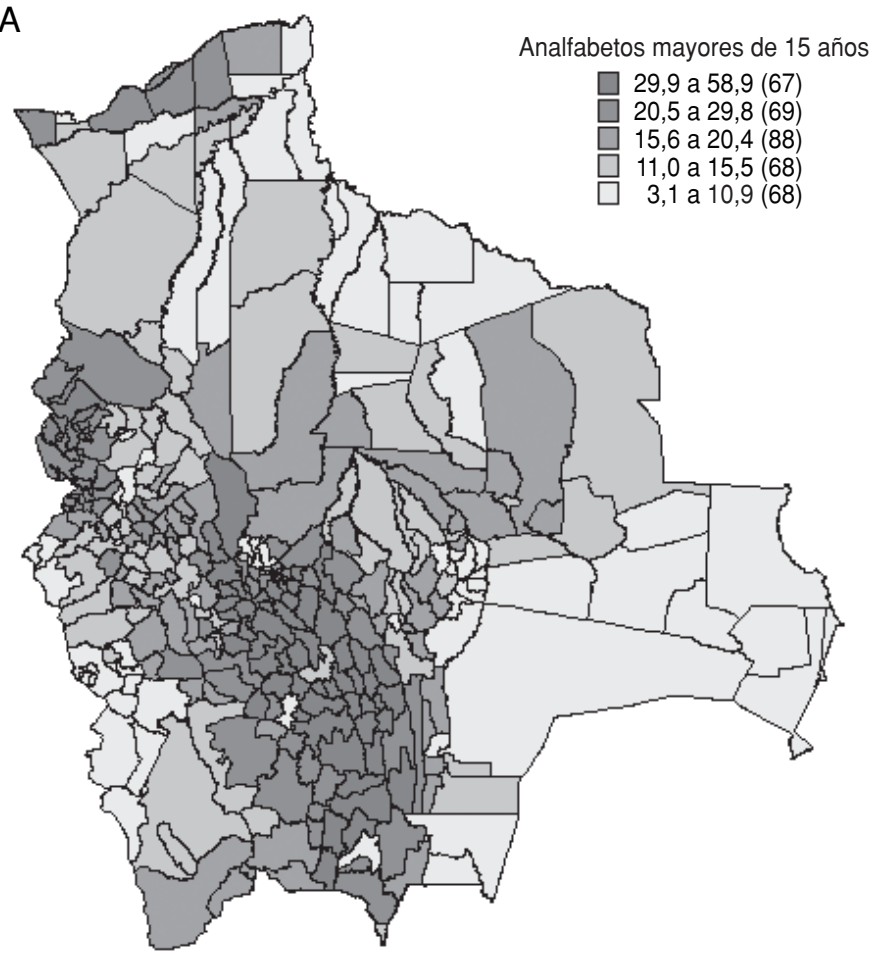

B

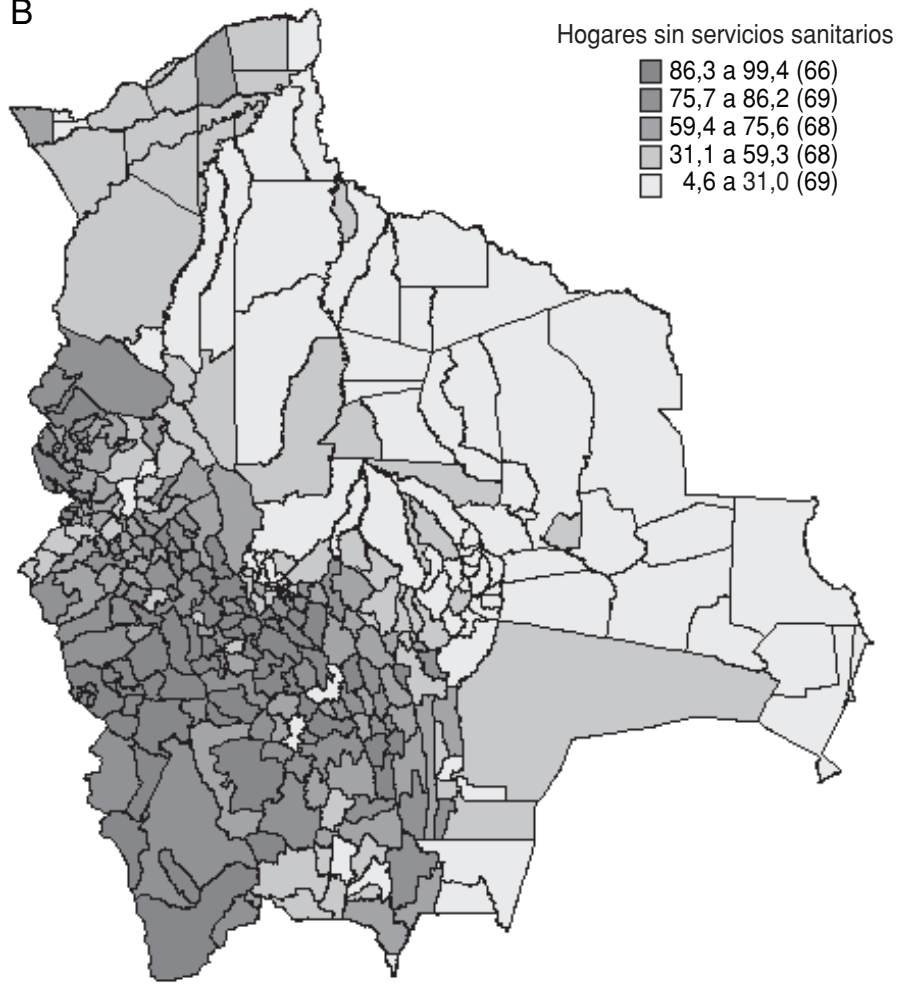

C

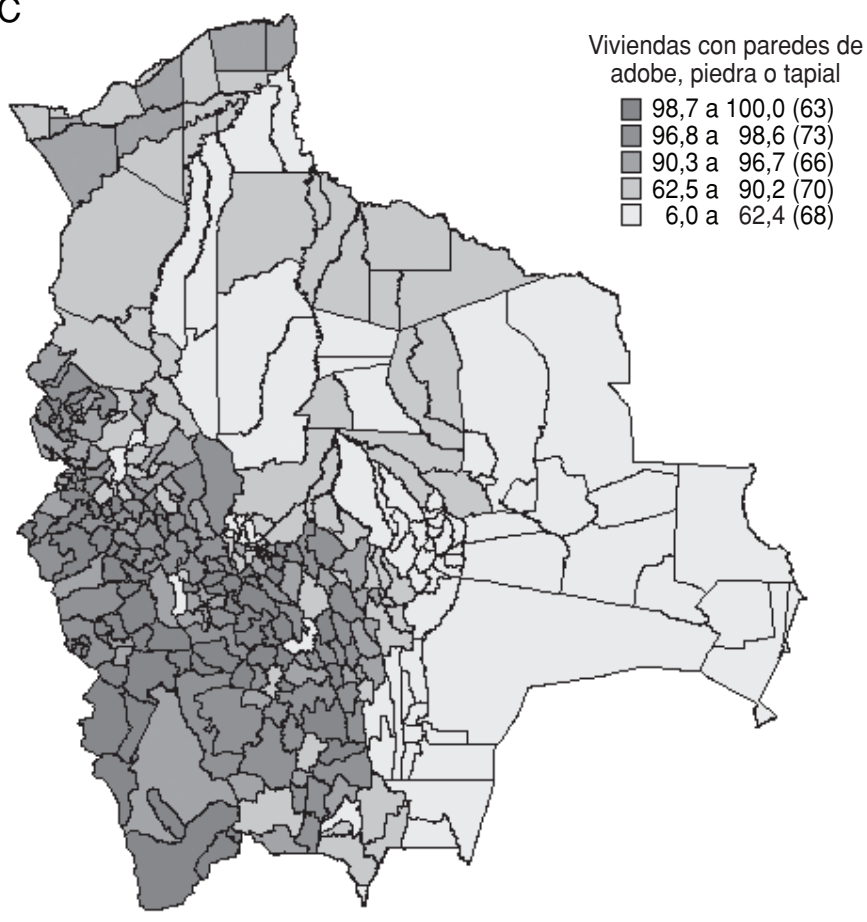

D

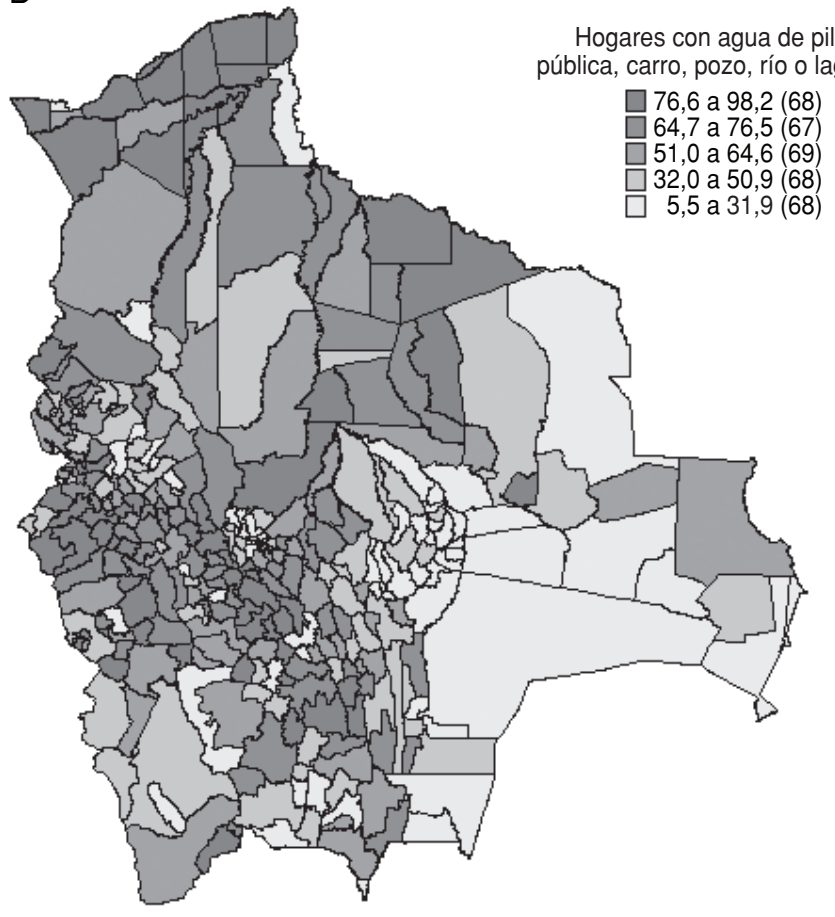

Fuente: a partir de los datos del Censo Nacional de Población y Vivienda de 2001 (9).

a En porcentajes del total. Entre paréntesis se ofrece el número de municipios clasificados en cada categoría. 
propiamente en los departamentos de Cochabamba, Chuquisaca, Potosí y el sur de La Paz (figura 3D). Además de los municipios con peores indicadores en general, se encontraron valores elevados en los municipios de la región nororiental del país, con cifras superiores a $51 \%$ en los departamentos del Beni y Pando. La brecha entre el valor mínimo (5,5\% en el municipio de Santa Cruz de la Sierra, departamento de Santa Cruz) y el máximo (98\% en el municipio Ingavi, departamento de Pando) se mantuvo muy amplia (cuadro 1), lo que se corrobora con los valores de los cuartiles 25 y 75 (35,1\% y $73,5 \%$, respectivamente).

El indicador "viviendas sin electricidad" mostró una mediana de $71,1 \%$ en los diferentes municipios (cuadro 1); el valor máximo de $100 \%$, registrado en el municipio de Coipasa, del departamento de Oruro, reflejó que hay municipios en los que la totalidad de las viviendas carecen de este servicio, mientras en el municipio de La Paz, del departamento del mismo nombre, esa proporción alcanzó apenas $4,7 \%$.

\section{Asociación entre la TMI y las variables socioeconómicas}

Si se superponen una por una las imágenes de los cuatro indicadores socioeconómicos graficados en la figura 3 y la distribución de la TMI mostrada en la figura 2 y se contrastan a su vez con la división político-administrativa de Bolivia (figura 1), se observarán distribuciones similares coincidentes con las áreas más marginadas del país en todos los casos, con un eje geográfico compuesto por La Paz, Oruro, Cochabamba, Chuquisaca y, especialmente, Potosí. Sin embargo, al analizar la correlación entre los indicadores mediante el coeficiente de Spearman (cuadro 2), se identificaron algunos indicadores cuyos altos valores de correlación con la TMI y entre sí sobresalen con respecto a los demás.

Como era de esperar, la variable "con agua fuera de la vivienda" presentó una alta correlación con la variable "con agua de pila, pozo o río" $(0,786)$, seguida de la alta correlación encontrada entre las variables "sin electricidad" y "con agua fuera de la vivienda" $(0,742)$ (cuadro 2). Esto indica que muchas viviendas con ese tipo de abastecimiento de agua carecían de energía eléctrica. Variables como "sin servicios sanitarios" y "con paredes de adobe piedra o tapial" (figuras 3B y 3C) mantuvieron una alta correlación entre sí $(0,768)$ y con la TMI $(0,684$ y 0,610 , respectivamente) (cuadro 2 ).

Los resultados obtenidos del ajuste de los modelos de regresión de Poisson (cuadro 3 modelo 1) muestran, en general, que al aumentar la privación socioeconómica aumentó la TMI. Las zonas con mayor porcentaje de población "sin servicios sanitarios" mostraron un mayor riesgo de mortalidad infantil $(\mathrm{RR}=1,94$; IC95\%: 1,82 a 2,07), seguidas de los municipios más afectados por la variable "con paredes de adobe, piedra o tapial" $(\mathrm{RR}=1,92$; IC95\%: 1,80 a 2,04). Asimismo, los municipios con mayor analfabetismo en mayores de 15 años tuvieron un riesgo mayor de mortalidad infantil (RR = 1,81; IC95\%: 1,71 a 1,91).

En los modelos multifactoriales ajustados por la variable "analfabetismo en mayores de 15 años" (cuadro 3, modelos 2 al $8)$, los indicadores sin servicios sanitarios ( $R R=1,50$; IC95\%: 1,38 a 1,66), con paredes de adobe, piedra o tapial $(\mathrm{RR}=1,54$;
IC95\%: 1,43 a 1,67$)$ y con techo de calamina, paja o palma ( $R R=1,34$; IC $95 \%$ : 1,26 a 1,43) mantuvieron una fuerte asociación con la TMI.

A mayor nivel de analfabetismo se observó un riesgo mayor de mortalidad infantil en el modelo 6 ( $R R=1,66$; IC95\%: 1,58 a 1,75), que también incluía la variable "con techo de calamina, paja o palma". Si se observa el riesgo relacionado con las demás variables ajustadas por el analfabetismo en mayores de 15 años, los municipios con mayor cantidad de viviendas con paredes de adobe, piedra o tapial presentaron también riesgos elevados de muerte infantil $(\mathrm{RR}=1,54$; IC95\%: 1,43 a 1,67), mayor que el correspondiente al analfabetismo solamente (RR = 1,34; IC95\%: 1,24 a 1,44) (cuadro 3, modelo 8).

En el modelo 7, el grupo 2 (ubicado entre los cuartiles 25 y 50) de la variable "con piso de cemento, tierra o ladrillo" presentó un riesgo de mortalidad infantil ( $R R=1,06$; IC95\%: 0,98 a 1,14) menor que con las variables restantes, aunque para el mismo grupo, el riesgo asociado con el mayor porcentaje de analfabetismo en ese modelo fue de $\mathrm{RR}=1,17$ (IC95\%: 1,08 a 1,26).

En general, los modelos ajustados pusieron en evidencia que al aumentar la privación socioeconómica aumentaba la TMI, tanto en los modelos con cada variable independiente por separado, como en los que además comprendían el analfabetismo en mayores de 15 años.

\section{DISCUSIÓN}

Este trabajo pone en evidencia la existencia de desigualdades en la mortalidad infantil en Bolivia, con mayores tasas en

CUADRO 2. Asociación entre los indicadores estudiados (coeficientes de correlación de Spearman), Bolivia, 2001

\begin{tabular}{|c|c|c|c|c|c|c|c|c|c|}
\hline Indicador & $\mathrm{TMI}^{\mathrm{a}}$ & $\begin{array}{c}\text { Analfabetismo } \\
\text { en mayores } \\
\text { de } 15 \\
\text { años }\end{array}$ & $\begin{array}{l}\text { Con agua } \\
\text { fuera de } \\
\text { la vivienda }\end{array}$ & $\begin{array}{l}\text { Con agua } \\
\text { de pila, } \\
\text { pozo o río }\end{array}$ & $\begin{array}{l}\text { Sin } \\
\text { electricidad }\end{array}$ & $\begin{array}{c}\text { Sin } \\
\text { servicios } \\
\text { sanitarios }\end{array}$ & $\begin{array}{c}\text { Con techo } \\
\text { de calamina, } \\
\text { paja } \\
\text { o palma }\end{array}$ & $\begin{array}{c}\text { Con piso } \\
\text { de cemento, } \\
\text { tierra o } \\
\text { ladrillo }\end{array}$ & $\begin{array}{c}\text { Con paredes } \\
\text { de adobe, } \\
\text { piedra o } \\
\text { tapial }\end{array}$ \\
\hline $\mathrm{TM}^{\mathrm{a}}$ & 1,0 & & & & & & & & \\
\hline Analfabetismo en mayores de 15 años & $0,467^{b}$ & 1,0 & & & & & & & \\
\hline Con agua fuera de la vivienda & $0,387^{b}$ & $0,185^{b}$ & 1,0 & & & & & & \\
\hline Con agua de pila, pozo o río & $0,474^{b}$ & $0,384^{b}$ & $0,786^{b}$ & 1,0 & & & & & \\
\hline Sin electricidad & $0,448^{b}$ & $0,355^{b}$ & $0,742^{b}$ & $0,635^{b}$ & 1,0 & & & & \\
\hline Sin servicios sanitarios & $0,684^{b}$ & $0,521^{b}$ & $0,484^{b}$ & $0,519^{b}$ & $0,526^{b}$ & 1,0 & & & \\
\hline Con techo de calamina, paja o palma & $0,435^{b}$ & $0,150^{\mathrm{b}}$ & $0,477^{b}$ & $0,389^{b}$ & $0,379^{b}$ & $0,543^{b}$ & 1,0 & & \\
\hline Con piso de cemento, tierra o ladrillo & $0,330^{\mathrm{b}}$ & $0,396^{b}$ & $0,398^{b}$ & $0,348^{b}$ & $0,513^{b}$ & $0,430^{b}$ & 0,1 & 1,0 & \\
\hline Con paredes de adobe, piedra o tapial & $0,610^{\mathrm{b}}$ & $0,439^{b}$ & $0,532^{b}$ & $0,497^{b}$ & $0,549^{b}$ & $0,768^{b}$ & $0,614^{b}$ & $0,499^{b}$ & 1,0 \\
\hline
\end{tabular}

a TMI: tasa de mortalidad infantil por 1000 nacidos vivos.

${ }^{b}$ Correlación significativa, $P \leq 0,01$. 
CUADRO 3. Asociación entre la tasa de mortalidad infantil y los indicadores estudiados, ${ }^{\text {a }}$ según el grupo de nivel socioeconómico, Bolivia, 2001

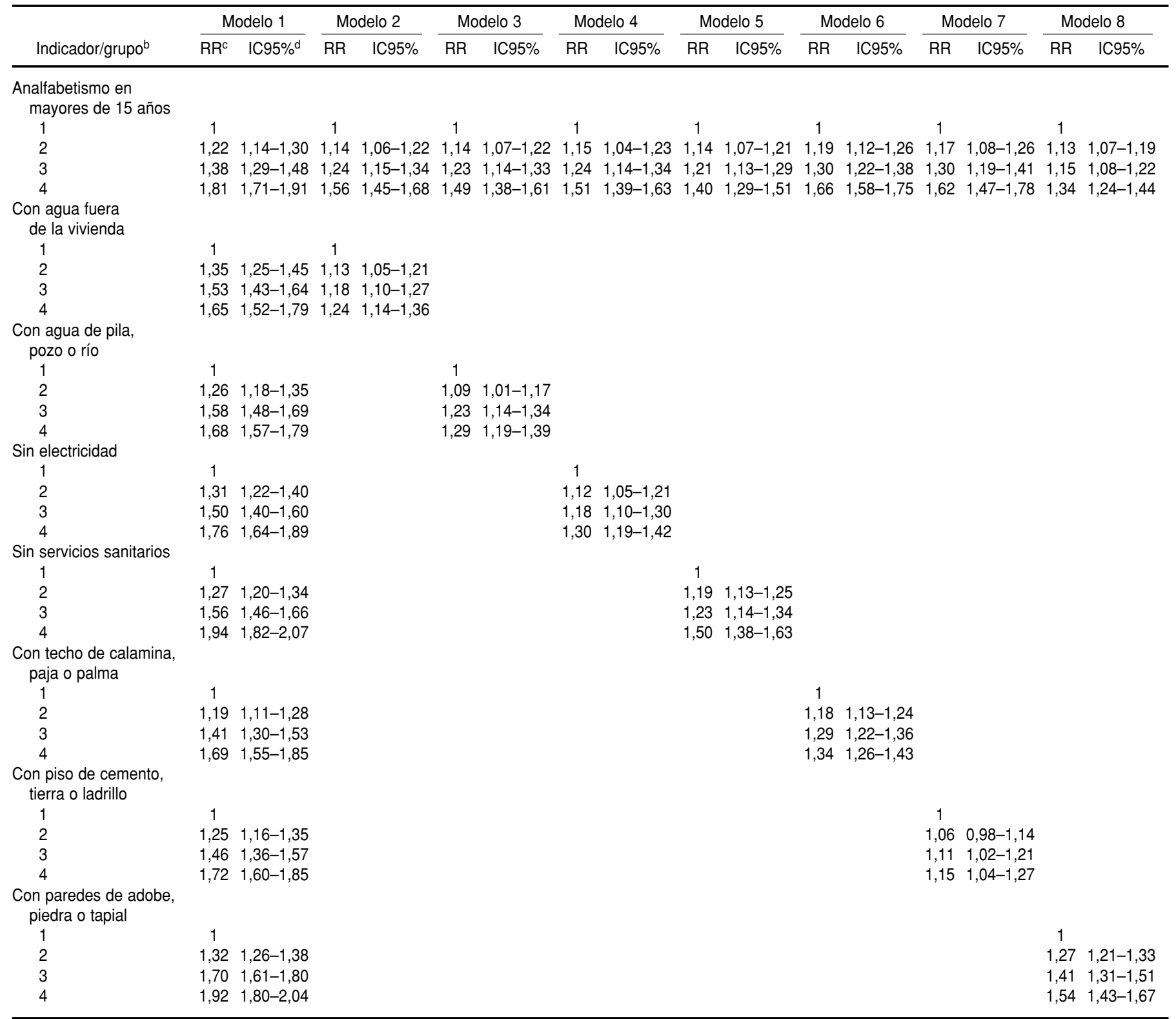

a Según el ajuste de ocho modelos de Poisson. Cada modelo contiene una de las variables independientes y la variable analfabetismo en mayores de 15 años, excepto el modelo 1 que contiene todas las variables.

b Los grupos se formaron a partir de los indicadores socioeconómicos de los municipios separados por los cuartiles: el grupo 1 corresponde al nivel inferior, por debajo del cuartil 25 , mientras que el grupo 4 corresponde a al nivel superior, por encima del cuartil 75.

${ }^{c} \mathrm{RR}$ : riesgo relativo.

d IC95\%: intervalo de confianza de $95 \%$.

los municipios de los departamentos Potosí, Oruro, La Paz y Cochabamba (altiplano y valle del sudoeste del país). Las menores tasas de mortalidad se encontraron en los municipios de los departamentos Santa Cruz, Beni, Tarija y Chuquisaca (valle y llano orientales). Estas desigualdades se corresponden con la inequidad socioeconómica, ya que los municipios con peores indicadores socioeconómicos presentaron mayores TMI.
Las desigualdades nacen de la desproporción en el acceso a diferentes tipos de recursos de algunos grupos sociales (18). Para algunos autores, las desigualdades sociales constituyen un obstáculo para mejorar la salud, el bienestar y el desarrollo social y económico de la población (19). Por otro lado, Breilh y colaboradores aseguran que las desigualdades inciden directamente sobre la situación de la salud de la población debido a las enor- mes brechas que se generan entre las clases sociales y las condiciones de trabajo, que a su vez condicionan la calidad de vida (20).

La mortalidad infantil no se debe solamente a la falta de asistencia médica, ya que el nivel de salud de la población, particularmente durante largos períodos, se ha asociado con el nivel socioeconómico y la disponibilidad de recursos. Este hecho explica la correlación encon- 
trada entre la TMI y el nivel de saneamiento básico, las condiciones generales de las viviendas y la educación, tal como se observa en el cuadro 2.

Según Vega y colaboradores, aunque la salud general ha mejorado en los últimos 15 años en Bolivia a la par que en toda América Latina, este cambio no ha ocurrido homogéneamente en los distintos grupos socioeconómicos del país. El mejoramiento de la salud infantil parece inclinarse de manera desproporcionada hacia los segmentos de la población que gozan de ventajas sociales y económicas, mientras que en los grupos más desfavorecidos esa mejora es menos sistemática y se produce a un ritmo mucho más moderado (21).

Otros estudios realizados en Brasil, Costa de Marfil, Filipinas, Ghana, Nepal, Nicaragua, Pakistán, Sudáfrica y Vietnam coinciden en que las desigualdades socioeconómicas afectan negativamente las TMI en los estratos menos favorecidos (22). En el caso de Bolivia, las desigualdades asociadas con la mortalidad infantil reflejan esa tendencia si se analizan los datos de forma global (a nivel nacional), sin embargo, a pesar de que las desigualdades socioeconómicas se reducen a nivel municipal (especialmente en municipios rurales), la mortalidad infantil se incrementa.

Organismos e instituciones internacionales aseguran que un gran número de las muertes en niños menores de 1 año son resultado de la desnutrición, las infecciones respiratorias agudas, la diarrea, el sarampión y la malaria o una combinación de estos factores y tienen como raíz la pobreza (23). Otros factores como la exclusión social, la falta de acceso a los servicios básicos (agua, electricidad y saneamiento), la precariedad laboral, la falta de articulación vial, las altas tasas de analfabetismo, junto con otros factores como la mala economía familiar, contribuirían al deterioro de la salud de esta población $(24,25)$.

Los resultados del presente estudio indican que una gran parte de los municipios que presentan mayor privación socioeconómica están habitados principalmente por personas de las etnias aimaras, quechuas, mocetenes, guaraníes y otros de la amazonia. Un estudio realizado en México en 1990 también detectó mayores desigualdades en los grupos indígenas (26).

Otro aspecto a tener en cuenta son las desigualdades existentes dentro de los municipios. Según el Banco Interamericano de Desarrollo, en Bolivia existen municipios en los que toda su población se encuentra en mala situación socioeconómica y las condiciones de vida precarias afectan de manera general a todos sus habitantes. Sin embargo, las desigualdades son escandalosamente visibles en los municipios más poblados y en las grandes ciudades, donde la brecha en las tasas de mortalidad es cada vez mayor (27). En ese sentido, el riesgo de muerte infantil en los municipios estudiados mantuvo patrones de comportamiento prácticamente similares en cuanto a la falta de agua y el saneamiento básico, el bajo nivel educacional y las condiciones que, en suma, son propias de las poblaciones aisladas, de difícil acceso y sin los beneficios de la cobertura de programas sanitarios preventivos o asistenciales.

Es evidente que la brecha en los niveles de desigualdad existentes a lo largo y ancho del territorio boliviano se amplía a medida que aumenta la migración de personas del campo hacia las grandes ciudades. Esto podría explicarse por la formación de conglomerados de familias pobres, marginadas y excluidas alrededor de las urbes, lo que hace más patente la cada vez mayor diferencia entre los pobres y los ricos. Estas diferencias se asocian negativamente con el nivel de inversión en el capital social y elevan las TMI (28).

Las desigualdades en la distribución de los ingresos están asociadas a las disparidades en el acceso a los servicios de salud - en particular la asistencia a los niños durante sus primeros años de vida- y la existencia de otros servicios sociales importantes, como vías de comunicación y medios de transportes. $\mathrm{La}$ falta de estos servicios contribuye a mantener los paupérrimos niveles de salud de la población y la elevada morbimortalidad existente en los países más pobres (29). En comparación con los niños de las familias más ricas, los niños de las familias pobres tienen entre la tercera parte y la mitad de las posibilidades de acceder a los servicios de salud y recibir tratamiento oportuno y eficaz contra muchas enfermedades, como las infecciones respiratorias agudas (30); al contrario, en los países desarrollados la población con menor nivel socioeconómico tiende a utilizar más los servicios de salud que la de mejor situación socioeconómica (31).

Uno de los factores determinantes que influyen en los sistemas de salud y la calidad y la utilización de los servicios a la población es la disponibilidad y calificación del personal sanitario que trabaja en los servicios locales de salud (32). Este factor es particularmente relevante en el altiplano y oriente bolivianos y en otras zonas pobres del país, donde el mayor problema es la accesibilidad a los servicios. Todo ello podría explicar las altas TMI en los municipios más alejados de las urbes más pobladas. En Bolivia, las grandes distancias entre las comunidades, comarcas o ayllus y los centros o postas sanitarias, así como la carencia de medios de transporte, la insuficiente infraestructura vial y los factores geográficos, climatológicos y culturales constituyen en ocasiones barreras infranqueables que impiden el acceso a los servicios de salud. El tiempo de viaje entre dos poblaciones puede ser de horas, días y hasta semanas (33).

Otros factores que pueden influir en las elevadas TMI encontradas en el presente trabajo son la falta de atención primaria y la insuficiente cobertura de vacunación completa. Bolivia presenta las peores cifras en estos indicadores en la Región de las Américas, el bajo nivel educativo de la población, la ausencia de saneamiento básico y la precariedad de sus viviendas son factores claves que triplican el riesgo de muerte (34).

\section{Fortalezas y limitaciones de este estudio}

En este trabajo se analiza por primera vez la distribución de las TMI en los departamentos de Bolivia, desagregada por los 327 municipios, y se asocia con las desigualdades socioeconómicas locales.

En este estudio se utilizaron los datos del Censo Nacional de 2001 para generar tanto las medidas de mortalidad como las variables socioeconómicas analizadas (35). Ante la ausencia de datos de mortalidad infantil, los datos obtenidos en el censo resuelven en parte este problema y el subregistro que prevalece en las estadísticas vitales en general; por su cobertura universal, los datos censales permitieron llegar a diferentes niveles de desagregación por áreas geográficas y grupos sociodemográficos.

Sin embargo, el área de menor desagregación fue el municipio y por lo tanto no se documentó la desigualdad existente al interior de los diferentes estratos socioeconómicos dentro de los municipios. El hecho de utilizar el analfabetismo en mayores de 15 años como indicador 
indirecto del nivel socioeconómico y, consecuentemente, del riesgo de muerte infantil, puede ser otra limitación de este estudio que se debe tener en cuenta al analizar sus conclusiones. No obstante, aunque existen otros indicadores indirectos, como el nivel de ingresos o la ocupación, algunos autores aseguran que la educación es un estimador más estable en el tiempo (36), ya que representa una ganancia permanente para el individuo.

\section{Conclusiones y recomendaciones}

Los resultados demostraron una fuerte asociación entre una mala situación socioeconómica y una elevada TMI en Bolivia en 2001. Los municipios de las zonas central y sudoriental del país presentaron la peor situación socioeconómica y las mayores TMI.

Se deben desarrollar mecanismos que aporten registros completos y confiables de los eventos de vida de la población y refinar las mediciones del Censo Nacional de Población y Vivienda dirigidas a las mujeres en edad fértil. Esto permitirá profundizar en el análisis de la situación de salud y optimizar las investigaciones. En esa línea, se insta a las autoridades nacionales y los organismos internacionales a perfeccionar las metodologías necesarias para generar estimados sólidos y sostenibles de la mortalidad infantil con un mayor nivel de desagregación en coordinación con los gobiernos estatales. Si bien la recolección de la información mediante encuestas demográficas y censos son fuentes secundarias importantes, se deben fortalecer los registros civiles como fuentes primarias para generar estimaciones precisas de las tendencias poblacionales y los eventos vitales.

Se recomienda realizar estudios más amplios y específicos del nivel de segre- gación socioeconómica y los niveles de escolaridad, especialmente en las grandes ciudades, ya que es donde se concentra la mayor parte de la población boliviana. Esos estudios deben enfocarse no solamente en el impacto de los factores socioeconómicos en el nivel de salud de los niños menores de 1 año, sino ampliarse a los niños menores de 5 años.

La información obtenida de este y otros estudios sobre desigualdades debe utilizarse tanto por los trabajadores de salud pública - para implementar intervenciones específicas destinadas a reducir las desigualdades y mejorar el nivel de acceso a los servicios de salud-como por los planificadores y tomadores de decisiones, encargados de generar e implementar políticas dirigidas a disminuir la exclusión social y formular mecanismos de redistribución económica, a fin de elevar el nivel de equidad social.

\section{REFERENCIAS}

1. Artazcoz L, Borrell C, Benach J, Bouso L, Cortès I, Fernández E, et al. Conceptes, antecedents $\mathrm{i}$ causes de les desigualtats socials en la salut. En: Borrell C, Benach J (coordinadores). Informe CAPS-Fundació Jaume Bofill. Les desigualtats en la salut a Catalunya. Barcelona: Editorial Mediterrània; 2003. Pp. 29-30.

2. Alazraqui M, Mota E, Spinelli H, Guevel C. Desigualdades en salud y desigualdades sociales: un abordaje epidemiológico en un municipio urbano de Argentina. Rev Panam Salud Publica. 2007;21(1):1-10.

3. Ferranti D, Perry F, Ferreira G, Walton M. Desigualdades en América Latina y el Caribe: ¿ruptura con la historia? Washington, D.C.: Banco Mundial; 2004

4. Unidad de Análisis de Políticas Sociales y Económicas, Instituto Nacional de Estadística, Banco Mundial. Pobreza y desigualdad en municipios de Bolivia: estimación del gasto de consumo combinando el Censo de 2001 y las encuestas de hogares. 2. ${ }^{\text {a }}$ ed. La Paz: UDAPE; 2006.

5. Heredia N. Equidad y determinantes sociales de la salud. 59. a Asamblea de la OMS. Ginebra: OMS; 2006. Hallado en http:// www.who.int/entity/social_determinants/ resources/bolivia.pdf. Acceso el 5 de marzo de 2009.

6. República de Bolivia, Ministerio de Salud y Deportes. Situación de salud, Bolivia 2004. La Paz: MSD; 2006. (Serie Documentos de Divulgación Científica BO3.1-3047-CNID.)

7. Instituto Nacional de Estadísticas. Bolivia: niveles, tendencias y diferenciales de la fe- cundidad; 2003. Hallado en http://www.ine. gov.bo/PDF/Fecundidad/Fecundidad0.pdf. Acceso el 10 de abril de 2009.

8. Instituto Nacional de Estadísticas. Censo de Población y Vivienda 2001. La Paz: INE; 2003. Hallado en http://www.ine.gov.bo/cgi-bin/ Redatam/RG4WebEngine.exe/PortalAc tion?\&MODE=MAIN\&BASE=TallCreac\& MAIN=WebServerMain.inl. Acceso el 10 de abril de 2009.

9. Cámara Nacional de Comercio. El ABC de los aportes a la seguridad social. Bol Legal (La Paz). 2004;13:1-4. Hallado en http://www. boliviacomercio.org.bo/CNC/Boletines/Leg al13.pdf. Acceso el 10 de abril de 2009.

10. Conferencia Interamericana de Seguridad Social. La seguridad social en Bolivia. México, D.F.: CISS; 2007. (Serie Documentos de Divulgación Científica BO3.1-537-CNID.)

11. Lavadenz F, Schab N, Straatman H. Redes públicas, descentralizadas y comunitarias de salud en Bolivia. Rev Panam Salud Publica. 2001;9(3):182-9.

12. Instituto Nacional de Estadística. Informe del Censo Nacional de Población y Vivienda Bolivia 2001. La Paz: INE; 2002.

13. Instituto Nacional de Estadística. Anuario estadístico 2001. La Paz: INE; 2002. Hallado en http://www.ine.gov.bo/PDF/Anuario 2001/Anuario_2001.pdf. Acceso el 23 de marzo de 2009.

14. SPSS for Windows. Chicago: SPSS Inc.; 2001.

15. Stata Corporation. Stata statistical software TX. College Station: StataCorp LP; 2003.

16. MapInfo Corporation. MapInfo Professional 7.8. New York: MapInfo Corp.; 2004
17. Tobías Garcés A. Extensión del modelo de regresión con el paquete estadístico STATA. Madrid: Universidad Carlos III; 2007.

18. Hollstein RD, Vega MJ, Carvajal BY. Socioeconomic level and infant mortality in Chile in the period 1985-1995. Rev Med Chile. 1998: 126(3):333-40.

19. Artazcoz L, Borrell C, Benach J, Bouso L, Cortès I, Fernández E, et al. Els eixos de la desigualtat. En: Borrell C, Benach J (coordinadores). Informe CAPS-Fundació Jaume Bofill. Evolució de les desigualtas en la salut a Catalunya. Barcelona: Editorial Mediterrània; 2005. Pp. 37-43.

20. Breilh J, Granada E, Campaña A, Betancourt OF. Social class and inequality regarding death in Quito. Quito: Ediciones Ilus; 1983.

21. Vega J, Hollstein RD, Delgado I, Marshall G, Yach D. Social inequalities and health in an intermediate-development nation: education and adult mortality in Chile, 1980-1996. In: Evans T, Whitehead M, Diderichsen F, Bhuiya A, Wirth M, ed. Challenging inequalities in health: from ethics to actions. New York: Oxford University Press; 2001. Pp. 123-37.

22. Wagstaff A. Socioeconomic inequalities in child mortality: comparisons across nine developing countries. Bull World Health Organ. 2000;78(1):19-29.

23. República de Bolivia, Ministerio de Previsión Social y Salud Pública. Causas de mortalidad hospitalaria en menores de 5 años, 1998-2000. La Paz: MPSSP; 2002.

24. Alleyne G, Castillo-Salgado C, Schneider MC, Loyola E, Vidaurre M. Overview of social 
inequalities in health in the Region of the Americas, using various methodological approaches. Rev Panam Salud Publica. 2002;12 (6):388-97.

25. Barbosa da Silva J, Berti M. Epidemiologia e desigualdades: notas sobre a teoria e a historia. Rev Panam Salud Publica. 2002;12(6): 375-83.

26. Bobadilla JL, Langer A. La mortalidad infantil en México: un fenómeno en transición. Rev Mex Sociol. 1990;52(1):111-31.

27. Inter-American Development Bank. Economic and social progress in Latin America, 1998-1999. Facing up to inequality in Latin America. Washington, D.C.: IABD; 1999.

28. Kawachi I, Kennedy BP, Lochner K, Prothrow-Stith D. Social capital, income inequality and mortality. Am J Public Health. 1997; 87:1491-514

29. Deaton A. Health, inequality, and economic development. New Jersey: Princeton University; 2001. Hallado en http:/ / www.rand. org/
labor/aging/rsi/Deaton-inequ-heal-all.pdf. Acceso el 23 de marzo de 2009.

30. Gwatkin DR, Rutstein S, Johnson K, Suliman A, Wagstaff A, Amozou A. Socio-economic differences in health, nutrition, and population within developing countries: an overview. Washington, D.C.: World Bank; 2007.

31. Fotso JC. Child health inequities in developing countries: differences across urban and rural areas. Int J Equity Health. 2006;5:9.

32. Lavy V, Strauss J, Thomas D, De Vreyer P. Quality of care, survival and health outcomes in Ghana. J Health Econ. 1996;15:333-57.

33. Wong E, Popkin B, Guilkey D, Akin J. Accessibility, quality of care and prenatal care use in the Philipines. Soc Sci Med. 1987;24: 927-44.

34. Darras C. Diferencias de mortalidad infantil dentro de Bolivia. Rev Panam Salud Publica. 1998;4(6):393-7. Hallado en http://www. scielosp.org/pdf/rpsp/v4n6/4n6a5.pdf. Acceso el 23 de marzo de 2009.
35. Alarcón D, Robles M. Retos para medir la mortalidad infantil con registros civiles incompletos. Washington, D.C.: Instituto Interamericano para el Desarrollo Social; 2007. Hallado en http://www.prenatal.tv/lecturas/ Medir\%20mortalidad\%20infantil.pdf. Acceso el 23 de marzo de 2009.

36. Vega J, Hollstein RD, Delgado I, Pérez K, Carrasco S, Marshall G, et al. Chile: socioeconomic differentials and mortality in a middle income nation. In: Evans T, Whitehead $\mathrm{M}$ Diderichsen F, Bhuiya A, Wirth M, ed. Challenging inequalities in health: from ethics to actions. Oxford: Oxford University Press. 2001. Pp. 133-47.

Manuscrito recibido el 3 de marzo de 2008. Aceptado para publicación, tras revisión, el 26 de noviembre de 2008.

ABSTRACT Objectives. To evaluate socioeconomic inequalities and its relation to infant mortality in Bolivia's municipalities in 2001.

\section{Socioeconomic inequalities and infant mortality in Bolivia}

Methods. An ecological study based on data from the 2001 National Census on Population and Housing (Censo Nacional de Población y Vivienda) covering the $327 \mathrm{mu}-$ nicipalities in Bolivia's nine departments. The dependent variable was the infant mortality rate (IMR); the independent variables were indirect socioeconomic indicators (the percentage of illiterates older than 15 years of age, and the building materials and sanitation features of the houses). The geographic distribution of each indicator was determined and the associations between IMR and each socioeconomic indicator were calculate using Spearman's rank correlation coefficient and adjusted with Poisson regression models.

Results. The resulting IMR for Bolivia in 2001 was 67 per 1000 live births. Rates ranged from < 0.1 per 1000 live births in the Magdalena municipality, Beni department, to 170.0 per 1000 live births in the Caripuyo municipality, Potosí department. The mean rate of illiteracy per municipality was $17.5 \%$; the mean percentage of houses without running water was $90.4 \%$, and for those lacking sanitation services, $67.6 \%$. The IMR was inversely associated with all of the socioeconomic indicators studied. The highest relative risk was found in housing without sanitation services. Multifactorial models adjusted for illiteracy showed that the following indicators were still strongly associated with the IMR: no sanitation services (Relative risk $(R R)=1.54 ; 95 \%$ Confidence Interval $(95 \% \mathrm{CI})=1.38-1.66)$; adobe, stone, or mud walls (RR = 1.54; 95\%CI: 1.43-1.67); and, corrugated metal, straw, or palm branch roof $(\mathrm{RR}=1.34 ; 95 \% \mathrm{CI}$ : $1.26-1.43)$.

Conclusions. A significant association was found between poor socioeconomic status and high IMR in Bolivia's municipalities in 2001. The municipalities in the country's central and southeastern areas had lower socioeconomic status and higher IMR. The lack of education, absence of basic sanitation, and precarious housing conditions were key factors that tripled the risk of death.

Key words Infant mortality, social inequity, social conditions, Bolivia. 